\title{
A GROUP VARIETY DEFINED BY A SEMIGROUP LAW
}

\author{
A. YU. OL'SHANSKII and A. STOROZHEV
}

(Received 27 June 94)

Communicated by J. R. J. Groves

\begin{abstract}
A group variety defined by one semigroup law in two variables is constructed and it is proved that its free group is not a periodic extension of a locally soluble group.
\end{abstract}

1991 Mathematics subject classification (Amer. Math. Soc.): 20E10, $20 \mathrm{F06.}$

In group theory a law in variables $x_{1}, x_{2}, \ldots, x_{n}$ is called a semigroup law if it can be represented in the form

$$
u_{1}\left(x_{1}, \ldots, x_{n}\right)=u_{2}\left(x_{1}, \ldots, x_{n}\right)
$$

where $u_{1}$ and $u_{2}$ are semigroup words, that is words which do not contain $x_{i}^{-1}$ for $i=1, \ldots, n$.

Obviously every group of finite exponent satisfies a nontrivial semigroup law. It is established in [4] that nilpotent groups of a given class can be defined by a semigroup law. Therefore free groups of a product of a locally nilpotent variety and a periodic variety satisfy a nontrivial semigroup law. As shown in [4], a nontrivial semigroup law follows from the property of being Engel. In [1], conditions under which soluble group varieties have a nontrivial semigroup law are studied. It is proved in [1] that a finitely generated soluble group satisfies a nontrivial semigroup law if and only if it has a nilpotent subgroup of finite index.

In view of these facts, one may raise a question concerning the existence of a finitely generated group with a semigroup law which is not a nilpotent-by-periodic group. In [2], the question of whether a 2-generated group without free subsemigroups must be

The work by the first author was partly supported by ISF, grant MID 000 , and by Russian Fundamental Research Foundation, grant 0111541.

(C) 1996 Australian Mathematical Society $0263-6115 / 95 \$ A 2.00+0.00$ 
a periodic extension of a locally nilpotent group is posed. In this paper the following theorem is proved.

THEOREM. There exists a nontrivial semigroup law such that some 2-generated group, which is not a periodic extension of a locally soluble group, satisfies this law.

Thus, in particular, the negative answer to the question raised in [2] is obtained.

To prove the Theorem, we introduce some word in two variables and then study a 2-generated relatively free group of the variety defined by the word.

We put

$$
\begin{gathered}
v=v(x, y)=x^{d} y^{d}, \\
w(x, y)=v^{n+1} x v^{n+4} x \cdots v^{n+(h-1)^{2}} x v^{n+h^{2}} x v^{-n-(h+1)^{2}} x^{-1} \cdots v^{-n-(2 h-1)^{2}} x^{-1} v^{-n+h^{2}(2 h-3)} x^{-1},
\end{gathered}
$$

where $h, d$ and $n$ are sufficiently large natural numbers. Note that both the sum of exponents of the word $v$ and that of the letter $x$ in the word $w(x, y)$ are equal to zero as the equation $1^{2}+2^{2}+\cdots+k^{2}=k(2 k+1)(k+1) / 6$ holds.

The study of the 2-generated relatively free group of the variety defined by the word $w(x, y)$ uses the technique, described in [3], of geometric interpretation for the deduction of consequences of defining relations. Following the patterns detailed in [3;25.1] and [3;29.3], we define groups $G(i)$ for every nonnegative integer $i$ and the group $G(\infty)$ with the corresponding alterations. We assume that the alphabet of presentations of these groups consists of the letters $a$ and $b$.

While [3] is the main source of information for references, in this paper we also use a few results obtained in [5].

LEMMA 1. Let $A$ be a simple word in rank $i$ or a period of rank $j \leq i$ and let some power $A^{f}$ of the word $A$ be conjugate in rank $i$ to the value $v(X, \bar{Y})$ for words $X$ and $\bar{Y}$ such that $w(X, \bar{Y}) \stackrel{i}{\neq} 1$. Then $1 \leq|f| \leq 100 \zeta^{-1}$.

Proof. Notice that the words $X$ and $\bar{Y}$ cannot be commutative in rank $i$ since otherwise the equation $w(X, \bar{Y}) \stackrel{i}{=} 1$ would hold.

Suppose that $f=0$, then $X^{d} \bar{Y}^{d} \stackrel{i}{=} 1$. Hence $X^{d}$ and $\bar{Y}^{d}$ commute in rank $i$ which implies the commutativity of $X$ and $\bar{Y}$ in rank $i$ by [3; Lemma 25.2] and [3; Lemma 25.12]. Therefore, $|f| \geq 1$.

Since the words $X$ and $\bar{Y}$ are not commutative in rank $i$, the inequality $|f| \leq 100 \zeta^{-1}$ holds by [5; Lemma 3].

LEMMA 2. In the notation of $[3 ; 30.2]$, we assume that $T$ is a word minimal in rank $i$ such that $T \stackrel{i}{=} W^{-1} X W$. Then $|T|<d|A|$. 
PROOF. The word $X^{d} \bar{Y}^{d}$ is conjugate to $A^{f}$ in rank $i$ and we can turn a conjugacy diagram of the words $X^{d} \bar{Y}^{d}$ and $A^{f}$ into a diagram $\Delta$ on a sphere with three holes and three cyclic segments $q_{1}, q_{2}, q_{3}$ of the contour with the labels $\varphi\left(q_{1}\right) \equiv C^{m}$, $\varphi\left(q_{2}\right) \equiv B^{k}, \varphi\left(q_{3}\right) \equiv A^{-f}$. By [3; Lemma 24.9] and [3; Lemma 22.2] applied to the diagram $\Delta$, we have $|Z|<2\left(\left|C^{m}\right|+\left|B^{k}\right|+\left|A^{f}\right|\right)$. Therefore, $|W|<\bar{\alpha}\left(\left|C^{m}\right|+\left|B^{k}\right|+\right.$ $\left.4\left(\left|C^{m}\right|+\left|B^{k}\right|+\left|A^{f}\right|\right)+\left|A^{f}\right|\right)<3\left(\left|C^{m}\right|+\left|B^{k}\right|+\left|A^{f}\right|\right)$ by [3; Lemma 25.4], hence $|T|<7\left(\left|C^{m}\right|+\left|B^{k}\right|+\left|A^{f}\right|\right)$. Assume that $|T| \geq d|A|$. Then $\left|C^{m}\right|+\left|B^{k}\right|>2 \zeta^{-2}\left|A^{f}\right|$. If $\left|B^{k}\right|<\zeta\left|C^{m}\right|$, then $\Delta$ is a $J$-map which is impossible by [5; Lemma 2] and [3; Lemma 25.8]. Hence $\left|B^{k}\right| \geq \zeta\left|C^{m}\right|$ and we can consider $\Delta$ as an $E$-map. By [3; Lemma 24.6] and [3; Lemma 25.10], the segments $q_{1}$ and $q_{2}$ of the contour of the diagram $\Delta$ are compatible, whence the commutativity of the words $X$ and $\bar{Y}$ in the rank $i$ follows, which contradicts the inequality $w(X, \bar{Y}) \stackrel{i}{\neq} 1$.

LEMMA 3. The word $T_{A, j}$ is not equal in rank $i$ to any power of the word $A$.

PROOF. If $T_{A, j}$ is conjugate to $A^{m}$ in rank $i$, then $X$ is conjugate to $A^{m}$ too, where $m \neq 0$. Therefore, by Lemma 1 , the diagram $\Delta$ considered in the proof of Lemma 2 is a $J$-map or an $E$-map, which is impossible by [5; Lemma 2] and [3; Lemma 25.8, Lemma 24.6, Lemma 25.10].

LEMMA 4. The presentation $G(\infty)$ satisfies condition $R 6$.

PROOF. It follows from the equations $A^{a_{1}} S A^{b_{1}} \stackrel{i-1}{=} A^{c_{1}} T A^{d_{1}}$ and $A^{a_{2}} S A^{b_{2}} \stackrel{i-1}{=}$ $A^{c_{2}} T A^{d_{2}}$ that $A^{a_{1}-c_{1}} S A^{b_{1}-d_{1}} \stackrel{i-1}{=} A^{a_{2}-c_{2}} S A^{b_{2}-d_{2}}$, which by [3; Lemma 25.18] and Lemma 3 implies the equations $a_{1}-c_{1}=a_{2}-c_{2}$ and $b_{1}-d_{1}=b_{2}-d_{2}$. Therefore, when proving that the presentation $G(\infty)$ satisfies condition $R 6$, we may consider equations $A^{a_{u}} S A^{b_{u}} \stackrel{i-1}{=} A^{c_{u}} T A^{d_{u}}$ where $u=1,2,3,4, c_{u}=a_{u}+p, d_{u}=b_{u}+q, S \equiv T_{A, j}^{ \pm 1}$, $T \equiv T_{A, t}^{ \pm 1}$ and the words $A^{a_{u}} S A^{b_{u}}$ and $A^{c_{u}} T A^{d_{u}}$ are consecutive subwords of cyclic shifts of the words $R_{A, j}$ and $R_{A, t}^{ \pm 1}$ such that

$$
\begin{aligned}
& b_{1}+a_{2}=(-1)^{r} f(A, j)\left(n+(k-1)^{2}\right), \\
& b_{2}+a_{3}=(-1)^{r} f(A, j)\left(n+k^{2}\right), \\
& b_{3}+a_{4}=(-1)^{r} f(A, j)\left(n+(k+1)^{2}\right)
\end{aligned}
$$

and

$$
\begin{aligned}
& d_{1}+c_{2}=(-1)^{s} f(A, t)\left(n+(m-1)^{2}\right), \\
& d_{2}+c_{3}=(-1)^{s} f(A, t)\left(n+m^{2}\right), \\
& d_{3}+c_{4}=(-1)^{s} f(A, t)\left(n+(m+1)^{2}\right)
\end{aligned}
$$


or

$$
\begin{aligned}
& d_{1}+c_{2}=(-1)^{s} f(A, t)\left(n+(m+1)^{2}\right), \\
& d_{2}+c_{3}=(-1)^{s} f(A, t)\left(n+m^{2}\right), \\
& d_{3}+c_{4}=(-1)^{s} f(A, t)\left(n+(m-1)^{2}\right)
\end{aligned}
$$

for some positive integers $k$ and $m$.

Then on one hand, for some number $M$ we have

$$
M=\frac{\left(b_{1}+a_{2}\right)-\left(b_{2}+a_{3}\right)}{\left(b_{2}+a_{3}\right)-\left(b_{3}+a_{4}\right)}=\frac{2 k-1}{2 k+1},
$$

and on the other hand,

$$
\begin{aligned}
M & =\frac{\left(b_{1}+q+a_{2}+p\right)-\left(b_{2}+q+a_{3}+p\right)}{\left(b_{2}+q+a_{3}+p\right)-\left(b_{3}+q+a_{4}+p\right)} \\
& =\frac{\left(d_{1}+c_{2}\right)-\left(d_{2}+c_{3}\right)}{\left(d_{2}+c_{3}\right)-\left(d_{3}+c_{4}\right)}=\left(\frac{2 m-1}{2 m+1}\right)^{ \pm 1}
\end{aligned}
$$

whence it follows that $k=m$ and the exponent of the expression $(2 m-1 / 2 m+1)^{ \pm 1}$ is equal to 1 . So the words $A^{c_{u}} T A^{d_{u}}$ are subwords of a cyclic shift of $R_{A, t}$ and not of $R_{A, t}^{-1}$. Hence

$$
\begin{aligned}
p+q & =\left(b_{1}+q+a_{2}+p\right)-\left(b_{1}+a_{2}\right) \\
& =\left(d_{1}+c_{2}\right)-\left(b_{1}+a_{2}\right) \\
& = \pm\left(\left(n+(k-1)^{2}\right) f(A, j)-\left(n+(k-1)^{2}\right) f(A, t)\right) \\
& = \pm\left(n+(k-1)^{2}\right)(f(A, j)-f(A, t)) .
\end{aligned}
$$

Similarly, $(p+q)= \pm\left(n+k^{2}\right)(f(A, j)-f(A, t))$. Therefore, $f(A, j)=f(A, t)$ and $p+q=0$. Thus $T \stackrel{i-1}{=} A^{-p} S A^{p}$ and the lemma is proved.

LEMMA 5. The presentation $G(i)$ satisfies condition $R 5$.

The proof of Lemma 5 is analogous to that of [3; Lemma 29.2].

LEMMA 6. The presentation $G(i)$ satisfies condition $R$.

The proof of Lemma 6 consists of references to the previous lemmas and to the definition of the presentation of $G(i)$.

LEMMA 7. The group $G(\infty)$ is a free group of the variety defined by the law $w(x, y) \equiv 1$. 
The proof of Lemma 7 is similar to that of [3; Theorem 19.7].

Now we can prove the Theorem.

PROOF. Let us assume that the group $G(\infty)$ is a periodic extension of a locally soluble group. Then $G(\infty)$ contains a nontrivial normal locally soluble subgroup $H$ as $G(\infty)$ is a torsionfree group by [3; Theorem 26.4]. Let $K$ be a 2-generated subgroup of $H$. Since $K$ is soluble, $K$ is abelian by [3; Lemma 25.14]. Hence $H$ is abelian. But any normal abelian subgroup of $G(\infty)$ is central by [3; Lemma 25.14]. Therefore, the subgroup generated by $H$ and $a$ is abelian and hence it is cyclic by [3; Theorem 26.5]. Since by [3; Lemma 25.12] if a nonzero power of some element of $G(\infty)$ is central, then this element is central too, the generator $a$ is central. Therefore, $[a, b] \stackrel{i}{=} 1$ for some $i$, which contradicts [3; Lemma 23.16], Lemma 6 and the definition of groups whose presentation satisfies condition $R$.

\section{References}

[1] J. A. Lewin and T. Lewin, 'Semigroup laws in varieties of solvable groups', Proc. Cambridge Philos. Soc. 65 (1969), 1-9.

[2] P. Longobardi, M. Maj and A. H. Rhemtulla, 'Groups with no free subsemigroups', Proc. Amer. Math. Soc., to appear.

[3] A. Yu. Ol'shanskii, Geometry of defining relations in groups, Mathematics and its applications volume 70 (Soviet Series) (Kluwer Academic Publishers, Dordrecht, 1991).

[4] A. I. Shirshov, 'On some positively defined group varieties', Sib. Mat. J. 8 (1967), 1190-1192.

[5] A. Storozhev, 'On abelian subgroups of relatively free groups', Comm. Algebra 22 (1994), 26772701.

Department of Mathematics

Moscow State University

Leninskie Gory, Moscow, 119899

Russia

e-mail: olsh@compnet.msu.su
Australian Mathematics Trust University of Canberra PO Box 1, Belconnen, ACT 2616

Australia

e-mail: ans@amt.canberra.edu.au 